Journal Club

Editor's Note: These short, critical reviews of recent papers in the Journal, written exclusively by graduate students or postdoctoral fellows, are intended to summarize the important findings of the paper and provide additional insight and commentary. For more information on the format and purpose of the Journal Club, please see http://www.jneurosci.org/misc/ifa_features.shtml.

\title{
Imaging Synaptic Vesicles Using VGLUT1-Venus Knock-In Mice: Insights into the Dynamic Nature of Intersynaptic Vesicle Exchange
}

\author{
Zahid Padamsey ${ }^{1}$ and Alexander Jeans ${ }^{1,2}$ \\ ${ }^{1}$ Department of Pharmacology, University of Oxford, Oxford, OX1 3QT, United Kingdom, and 2Department of Physiology, Anatomy and Genetics, \\ University of Oxford, Oxford, OX1 3QX, United Kingdom \\ Review of Herzog et al.
}

Traditionally, synapses have been regarded as compartmentalized nodes of communication, in which axonal boutons and dendritic spines operate independently of their neighbors and the efficacy of neurotransmission is regulated solely by patterned activity between presynaptic and postsynaptic cells. However, this notion has been challenged by several recent studies, which demonstrate that neighboring boutons and spines within a cell share resources over a varied timescale (minutes to hours) and may not, therefore, function in a strictly independent manner (Staras, 2007; Staras et al., 2010).

Several resources, including proteins, nucleic acids, lipids, and organelles, have been shown to be exchanged between adjacent synapses, some of which are directly involved in synaptic communication. By photobleaching FM dye-labeled synapses in cultured neurons, Darcy et al. (2006) demonstrated that a fraction of synaptic vesicles originating from the recycling pool constitutively exchanges be-

Received Dec. 23, 2011; revised Jan. 20, 2012; accepted Jan. 20, 2012

This work was supported by the Natural Sciences and Engineering Research Council of Canada (to Z.P.), the Clarendon Scholarship (University of Oxford; to Z.P.), and the Medical Research Council (UK; to A.J.).

Correspondence should be addressed to either Zahid Padamsey or Alexander Jeans, Department of Pharmacology, University of Oxford, Mansfield Road, Oxford, OX1 3QT, United Kingdom. E-mail: zahid.padamsey@ pharm.ox.ac.uk or alexander.jeans@dpag.ox.ac.uk.

DOI:10.1523/JNEUROSCI.6425-11.2012

Copyright $\odot 2012$ the authors $\quad 0270-6474 / 12 / 323284-03 \$ 15.00 / 0$ tween neighboring presynaptic terminals. This observation gave rise to the concept of the synaptic vesicle "superpool," a shared pool of vesicles from several adjacent terminals that can be rapidly and directly accessed by individual synapses (Staras et al., 2010). Recently, a paper by Herzog et al. (2011) in The Journal of Neuroscience examined intersynaptic vesicle exchange both in vitro and in vivo, using mice expressing a knock-in vesicular glutamate transporter (VGLUT1) fused to the YFP variant Venus.

VGLUT1 localizes to synaptic vesicles, and its expression level within axonal boutons has been shown to accurately report synaptic vesicle number and influence presynaptic strength (see Herzog et al., 2006, 2011). To enable live imaging of synaptic vesicles, Herzog et al. (2011) generated a mouse in which the endogenous VGLUT1 locus was modified to encode a VGLUT1-Venus fusion protein. Because the Venus tag does not impair VGLUT1 function and endogenous levels of VGLUT1 expression are maintained by the knock-in approach, mice exhibited normal vesicular glutamate uptake and neurotransmitter release. Thus, the authors could study synaptic vesicle trafficking and function under physiological conditions without the potential confounding effects associated with either the overexpression of genetic reporters or the ad- ministration of exogenous labels such as FM dyes.

Herzog et al. (2011) examined intersynaptic vesicle trafficking by monitoring fluorescence recovery after photobleaching (FRAP) at individual terminals in VGLUT1-Venus mice in hippocampal neuronal cultures. Consistent with previous studies, fluorescence recovery was best described as a biexponential function with two time constants: an early, fast component $\left(\tau_{\mathrm{F}}=44.0 \mathrm{~s}\right)$, presumably reflecting rapid axonal transport of unbleached vesicles to the terminal, and a later component $\left(\tau_{\mathrm{S}}=28.5 \mathrm{~min}\right)$, likely mediated by the slower exchange of vesicles with neighboring, unbleached boutons (Darcy et al., 2006; Staras et al., 2010). Fluorescence recovery was never complete, and reached $\sim 46 \%$ after $1 \mathrm{~h}$, suggesting that approximately half of all synaptic vesicles were constitutively exchanged over this time.

Although confirming previous findings has value in itself, the main advantage of the VGLUT1-Venus line lies in its suitability for in vivo studies. To this end, the authors used two-photon laser scanning microscopy to determine characteristics of synaptic vesicle turnover by FRAP imaging of intact cortex in anesthetized mice. Several quantitative differences between presynaptic terminals in culture and in vivo emerged, the most surprising being that synaptic vesicle trafficking in 


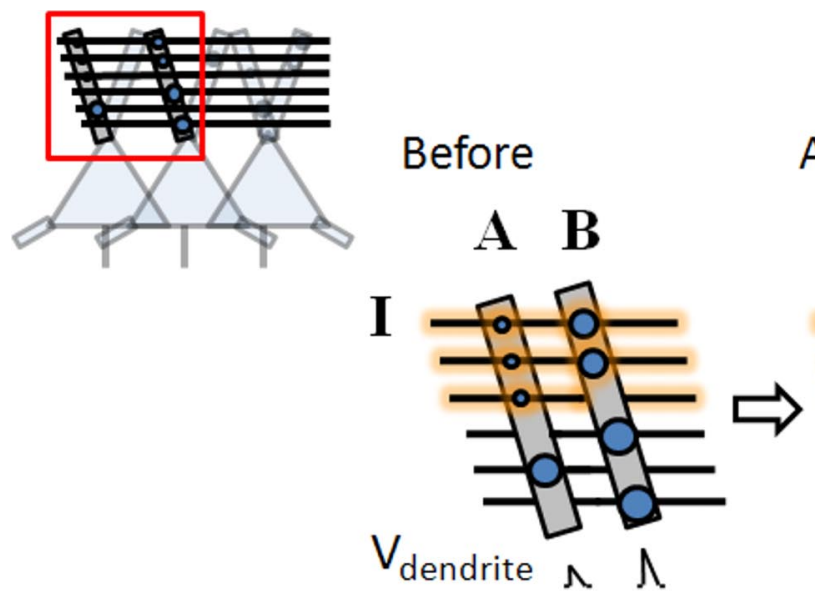

After
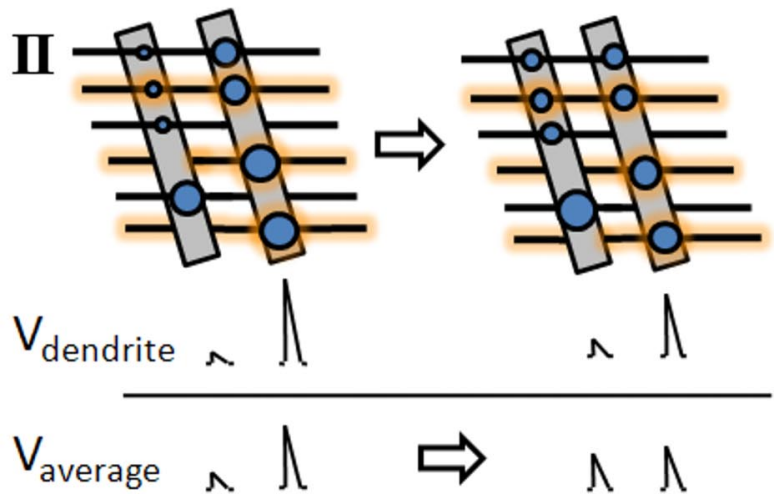

$\Lambda$

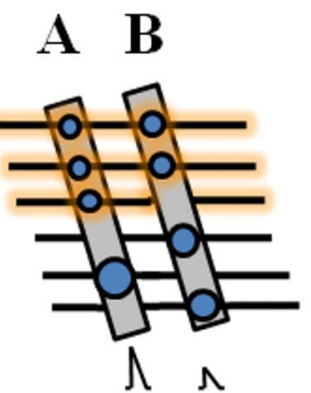

Figure 1. Intersynaptic vesicle exchange may improve pattern discrimination in dendritic networks. The responses of two dendritic processing units $(\mathbf{A}, \mathbf{B})$ to two different input patterns $(I, I)$ are shown $\left(V_{\text {dendrite }}\right)$. In these diagrams, presynaptic terminal size represents synaptic efficacy; active axons are highlighted in orange. Initially, both input patterns elicit relatively strong responses in $\mathbf{B}$, relatively weak responses in $\mathbf{A}$, and moderate responses in surrounding units (not shown), with which vesicles may also be exchanged. Note that the similarity of the qualitative response to both patterns means that the ability of the local dendritic network to distinguish between the two is limited. As a result of dendritic responses to inputs I and II, average levels of activity over time ( $\left.V_{\text {average }}\right)$ in $\mathbf{B}$ are greater than in its neighboring units, and average levels of activity in $\mathbf{A}$ are weaker than in its neighboring units. The resulting difference in activity between dendritic units drives the redistribution of vesicles between terminals associated with $\mathbf{A}, \mathbf{B}$, and surrounding units such that transmitter release is enhanced at boutons contacting less active units (A) and diminished at boutons contacting more active units (B). Although the changes at a given terminal may be modest, the cumulative effect of changes across terminals can significantly alter responses to patterned input. As a result of vesicle redistribution, input I now generates a relatively higher response in $\mathbf{A}$ than in $\mathbf{B}$; input II continues to generate a relatively higher response in $\mathbf{B}$ than in $\mathbf{A}$. The responses of the network to both patterns of input, which were initially highly correlated, have now become more distinct. Thus, the activity-dependent redistribution of input may improve pattern discrimination in dendritic networks.

vivo was significantly slower, indicated by slower kinetics for both phases of fluorescence recovery $\left(\tau_{\mathrm{F}}=220 \mathrm{~s}\right.$ vs $44.0 \mathrm{~s} ; \tau_{\mathrm{S}}=$ $44.5 \mathrm{~min}$ vs $28.5 \mathrm{~min}$ ). Presynaptic terminals examined in vivo also had a smaller proportion of exchanging vesicles (34\% vs $46 \%$ ) over the $1 \mathrm{~h}$ observation period.

What might account for these unexpected differences? The authors suggest a few possible explanations, including the different cell types (cortical vs hippocampal) and different developmental stages studied, as well as the effects of glia, neuromodulatory inputs, and general anesthetics on synapses in vivo. Despite these differences, it is clear that vesicle exchange was qualitatively similar in the two systems, and the same mechanisms appear to operate, albeit on different timescales. By allowing comparison between in vivo and in vitro conditions, these experiments therefore give some reassurance that in vitro cell culture models are likely to be reasonably representative of presynaptic function in vivo.

Although it is well established that synaptic vesicles exchange between boutons, the function of this exchange remains unclear. In their final set of experiments, Herzog et al. (2011) again followed individual presynaptic terminals in culture over $1 \mathrm{~h}$. Motivated by their earlier observation of apparently sudden, marked fluorescence changes at many boutons, they used much higher image-acquisition rates to record fluctuations more accurately.
The results clearly demonstrate a dynamic redistribution of synaptic vesicles across individual terminals: $32 \%$ of boutons showed a net increase in fluorescence over the hour, with an average rate of change of $0.5 \% / \mathrm{min} ; 29 \%$ showed a net decrease at the same average rate; and 39\% showed no net change (Herzog et al., 2011; their Fig. $8 E$ ). Furthermore, when small groups of adjacent boutons were analyzed independently, total fluorescence across the terminals was constant at any point in time, although individual boutons within the group could show substantial changes (Herzog et al., 2011; their Fig. 8C). This apparent conservation of synaptic vesicle numbers across clusters of terminals not only supports the superpool concept, but suggests that a gain of synaptic vesicles by one terminal must be balanced by a loss of equal magnitude at one or more nearby terminals on the same axon. Because exchanged vesicles enter the recycling pool (the fraction of vesicles at the synapse that is release-competent under physiological conditions) (Darcy et al., 2006), vesicle sharing might have wider implications for synaptic function and, more generally, information processing in neuronal networks.

\section{Can synaptic vesicle sharing promote} more efficient information processing? The structural properties of axons have been proposed to facilitate the dispersion of information in a network (Yuste, 2011), but could active processes operating within axons play a more active and dynamic role in distributing input? As a general principle, information processing should be as evenly distributed as possible among the available computational units to optimize processing efficiency. Excessive use or neglect of any of the processors may reduce the network's ability to decorrelate similar streams of information, since a processor that is frequently or infrequently activated is less useful for discriminating inputs. Dendritic branches have been proposed as a basic computational unit within the CNS (London and Häusser, 2005); a mechanism that apportions information flow as evenly as possible among groups of dendrites would therefore be highly desirable. Individual dendritic branches are preferentially activated by particular spatiotemporal patterns of synaptic input (Losonczy and Magee, 2006; Branco et al., 2010); however, circuit level activity within the brain undergoes constant experience-dependent remodeling, and the frequency of occurrence of favored patterns of input for any 
dendrite may change accordingly. Under these circumstances, an active means of redistributing information processing, perhaps by redirecting the flow of information away from highly active units to relatively less active units, would be advantageous; such redirection might be achieved by synaptic vesicle trafficking across boutons.

The size of the recycling pool at a given synapse is regulated in part by local levels of depolarization in the partner dendrite; in this way, presynaptic function is suppressed by a chronic elevation in dendritic depolarization and enhanced by a chronic reduction in dendritic activity (Branco et al., 2008; Jakawich et al., 2010). If intersynaptic vesicle exchange were to support this activity-driven regulation, in part at least, and given the conservation of vesicle numbers across clusters of neighboring synapses revealed by Herzog et al. (2011), then we would expect the recycling pool size, and thus presynaptic strength, at any given bouton to depend on the relative difference in activity between its associated dendrite and the dendrites associated with neighboring terminals. This would tend to minimize differences in the probability of activation among small groups of dendrites, thus ensuring that processing workload is distributed as evenly as possible, at least at a local level, and that computational efficiency is maintained (Fig. 1). In contrast to homeostatic regu- lation of presynaptic function, which is thought to rely on absolute levels of dendritic activity, this mechanism would exploit relative local differences to ensure that information processing is optimized.

In conclusion, the study by Herzog et al. (2011) contributes to a growing body of literature that suggests that synapses, rather than being functionally isolated from their neighbors, may in part be regulated by processes that operate across groups of synapses. From a physiological and perhaps even from a computational standpoint, such ensemble regulation may play an important role in maintaining optimal synaptic function. However, these studies are in their infancy, and an improved understanding of this regulatory crosstalk between synapses is likely to be an important goal over the coming years. Appropriate tools will be critical in enabling this work and, to this end, the work of Herzog et al. (2011) introduces a model animal that circumvents many of the potential confounds present in alternative approaches. The initial vesicle trafficking studies reported by Herzog et al. (2011) contribute important observations that are likely to be the first of many derived from this invaluable resource.

\section{References}

Branco T, Staras K, Darcy KJ, Goda Y (2008) Local dendritic activity sets release probability at hippocampal synapses. Neuron 59:475-485.

Branco T, Clark BA, Häusser M (2010) Den- dritic discrimination of temporal input sequences in cortical neurons. Science 329:1671-1675.

Darcy KJ, Staras K, Collinson LM, Goda Y (2006) Constitutive sharing of recycling synaptic vesicles between presynaptic boutons. Nat Neurosci 9:315-321.

Herzog E, Takamori S, Jahn R, Brose N, Wojcik SM (2006) Synaptic and vesicular co-localization of the glutamate transporters VGLUT1 and VGLUT2 in the mouse hippocampus. J Neurochem 99:1011-1018.

Herzog E, Nadrigny F, Silm K, Biesemann C, Helling I, Bersot T, Steffens H, Schwartzmann R, Nägerl UV, El Mestikawy S, Rhee J, Kirchhoff F, Brose N (2011) In vivo imaging of intersynaptic vesicle exchange using VGLUT1venus knock-in mice. J Neurosci 31:15544-15559.

Jakawich SK, Nasser HB, Strong MJ, McCartney AJ, Perez AS, Rakesh N, Carruthers CJ, Sutton MA (2010) Local presynaptic activity gates homeostatic changes in presynaptic function driven by dendritic BDNF synthesis. Neuron 68:1143-1158.

London M, Häusser M (2005) Dendritic computation. Annu Rev Neurosci 28:503-532.

Losonczy A, Magee JC (2006) Integrative properties of radial oblique dendrites in hippocampal CA1 pyramidal neurons. Neuron 50:291-307.

Staras K (2007) Share and share alike: trading of presynaptic elements between central synapses. Trends Neurosci 30:292-298.

Staras K, Branco T, Burden JJ, Pozo K, Darcy K, Marra V, Ratnayaka A, Goda Y (2010) A vesicle superpool spans multiple presynaptic terminals in hippocampal neurons. Neuron 66:37-44.

Yuste R (2011) Dendritic spines and distributed circuits. Neuron 71:772-781. 